\title{
Efficiency and fairness of new-additive increase multiplicative decrease congestion avoidance and control algorithm
}

\begin{abstract}
When using of the Internet increased dramatically; the congestion avoidance problem became even more important. The congestion is usually caused by the multiplexing for packets when the packets are at the bottleneck links. Efficiency and fairness are the important metrics in the performance of congestion avoidance mechanisms. And also all of the researches for the congestion avoidance algorithms, interest about this parameters metrics to evaluate the performance of the algorithms. This research studied the performance of the New-Additive Increase Multiplicative Decrease (AIMD) algorithm as one of the core protocols for the TCP congestion avoidance and control mechanism. In addition, to evaluate the effect of using the AIMD algorithm after its development to measure the efficiency and fairness and find new enhancement results for our approach, which named as the New-AIMD algorithm. The NCTUns simulator is used to obtain the results after implementing the modifications to the mechanism.
\end{abstract}

Keyword: AIMD algorithm; Congestion avoidance and control 